\title{
A Multivariable Analysis of Sedation, Activity, and Agitation in Critically Ill Patients using the Riker Scale, ECG, Blood Pressure, and Respiratory Rate
}

\author{
BA Janz ${ }^{1,2}$, GD Clifford ${ }^{1}$, JE Mietus ${ }^{2}$, RG Mark ${ }^{1,2}$ \\ ${ }^{1}$ Harvard-MIT Division of Health Sciences \& Technology, Cambridge MA, USA \\ ${ }^{2}$ Beth Israel Deaconess Medical Center, Boston MA, USA
}

\begin{abstract}
Patients that are over-sedated or dangerously agitated in the Intensive Care Unit (ICU) have longer lengths of stays, and poorer outcomes in the ICU. The authors have evaluated heart rate (HR), blood pressure (BP) and respiratory rate $(R R)$ in 2938 patients with 124,591 separate Riker (agitation/sedation) class scores over a three year period. The mean and standard deviation for $H R, B P$ and $R R$ in each Riker class were calculated together with inter-class $F$ - and t-tests. Although there was a statistically significant difference in $H R, B P$ and $R R$ between each class except class 6 and class 7 , empirical estimates of the joint probability density functions demonstrate that class overlap is overwhelming. This overlap essentially prevents individual Riker classification using $H R, B P$ and $R R$. In order to improve this classification, an ECG-derived noise metric was evaluated on sections of an ECG corresponding to Riker values of 1, 4 and 7, with promising results.
\end{abstract}

\section{Introduction}

The overall goal of this project is to define physiologic variables in the ICU that can help quantify sedation, agitation, and activity. This study was designed to look specifically at agitation and sedation using the Riker scale, heart rate (HR), blood pressure (BP), and respiratory rate (RR) in critically ill patients. To help further differentiate agitation, the activity of a patient was evaluated using an ECG derived noise metric or 'noisy beat' algorithm.

Due to the fact that most of these patients are critically ill and a large percentage of patients require ventilatory support, sedation is used to help keep the patient comfortable. Sedation can be subjectively measured by the ICU staff using methods such as the Riker scale[1]. Agitated patients can harm themselves or can be oversedated as a result of their increased activity, leading to an increase in morbidity and mortality [2]. The sedation and agitation of a patient can also be related to the sleep quality, pain and mental status of a patient (Figure 1).

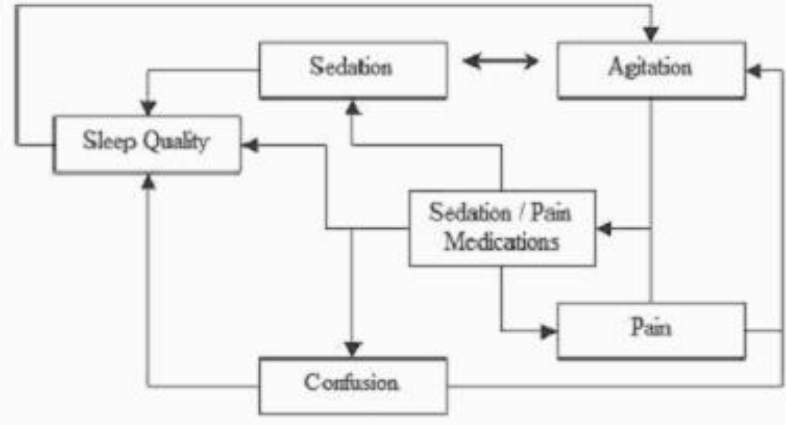

Figure 1 The Pain, Agitation, Activity, Sleep and Sedation (PAASS) Cycle

There are several different methods to differentiate sedation and agitation including subjective measures such as the Riker scale and more objective measures such as evaluating blood pressure, heart rate variability, or digital imagery [2, 3]. The Riker Scale (score) is one method to evaluate critically ill patients in the ICU and is based on classifying patients numerically (Table 1). A class score of one corresponds to an unarousable patient and a seven corresponds with a dangerously agitated patient [1]. The score is a subjective account of the overall state of the patient and might vary depending on the nurse caring for the patient or local interpretation of the score (scale).

\begin{tabular}{|c|l|l|}
\hline$\#$ & \multicolumn{1}{|c|}{ Riker } & \multicolumn{1}{c|}{ Description } \\
\hline 1 & Unarousable & Minimal or no response to noxious stimilu \\
\hline 2 & Very Sedated & $\begin{array}{l}\text { Arouses to physical stimuli but does not } \\
\text { communicate or follow commands }\end{array}$ \\
\hline 3 & Sedate & $\begin{array}{l}\text { Difficult to arouse but awakens to verbal } \\
\text { stimuli or gentle shaking }\end{array}$ \\
\hline 4 & Calm/Cooperative & $\begin{array}{l}\text { Follows commands, easily arousable, } \\
\text { Calm }\end{array}$ \\
\hline 5 & Agitated & $\begin{array}{l}\text { Anxious or physically agitated, calms to } \\
\text { verbal instructions }\end{array}$ \\
\hline 6 & Very agitated & $\begin{array}{l}\text { Requiring restraint and frequent verbal } \\
\text { reminding of limits, biting ETT }\end{array}$ \\
\hline 7 & $\begin{array}{l}\text { Dangerously } \\
\text { agitated }\end{array}$ & $\begin{array}{l}\text { Pulling at ET tube, trying to remove } \\
\text { catheters, climbing over bedrail, striking at } \\
\text { staff, thrashing side-to-side }\end{array}$ \\
\hline
\end{tabular}

Table 1 Riker Class Scores (Scale Values) [1] 


\section{Methods}

Patient information, physiologic parameters, and the Riker score data were extracted from a large critical care research database (MIMIC II) [4]. Random patients from the database were analyzed to evaluate the relationship between different Riker classes and physiologic values. The machine derived and nurse verified heart rate, blood pressure, and respiratory rate were selected using a SQL command for every Riker value entered for each patient in the Oracle ${ }^{\mathrm{TM}}$ database. The mean and standard deviation for each Riker class (17) was evaluated with the student's t-test using MatLab ${ }^{\mathrm{TM}}$.

Five additional patients were then evaluated with an ECG-derived noise metric (noise index) to distinguish agitated patients from calm and sedate patients. The hypothesis is that agitated patients will be more active than sedate or calm patients and will have noisier ECG rhythm. Patients that had a Riker score of 1, 2, 4, 5, and 7 during an ICU admission were evaluated using the ECG derived noise metric [5].

\section{Results}

2,938 patients were randomly selected from the MIMIC II database. The average ICU length of stay was 2.93 days. There were a total of 124,591 separate Riker values entries (42.4 entries per patient). Figure 2 shows the distribution of entered values for each Riker class.

\begin{tabular}{|c|c|c|c|c|c|}
\hline 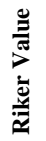 & 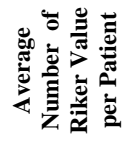 & 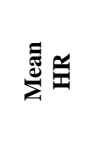 & 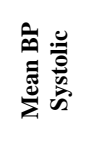 & 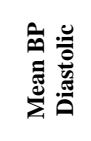 & 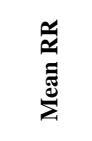 \\
\hline 1 & 18.1 & $\begin{array}{c}90.4 \\
+/-17.1\end{array}$ & $\begin{array}{c}122.8 \\
+/- \\
23.0 \\
\end{array}$ & $\begin{array}{c}58.7 \\
+/-12.6\end{array}$ & $\begin{array}{c}22.4 \\
+/-9.0\end{array}$ \\
\hline 2 & 8.0 & $\begin{array}{r}89.8 \\
+/-16.6 \\
\end{array}$ & $\begin{array}{c}116.3 \\
+/-20.2 \\
\end{array}$ & $\begin{array}{c}56.1 \\
+/-12.3 \\
\end{array}$ & $\begin{array}{c}20.3 \\
+/-7.6\end{array}$ \\
\hline 3 & 23.6 & $\begin{array}{c}87.2 \\
+/-17 \\
\end{array}$ & $\begin{array}{c}120.2 \\
+/-21.6 \\
\end{array}$ & $\begin{array}{c}58.5 \\
+/-13.5 \\
\end{array}$ & $\begin{array}{c}19.9 \\
+/-19.9 \\
\end{array}$ \\
\hline 4 & 56.6 & $\begin{array}{c}85.1 \\
+/-15.8 \\
\end{array}$ & $\begin{array}{c}122.8 \\
+/-22.3 \\
\end{array}$ & $\begin{array}{c}57.7 \\
+/-14.4 \\
\end{array}$ & $\begin{array}{c}21.1 \\
+/-6.3 \\
\end{array}$ \\
\hline 5 & 10.4 & $\begin{array}{c}93.5 \\
+/-17.9\end{array}$ & $\begin{array}{c}133.2 \\
+/-25.6\end{array}$ & $\begin{array}{c}63.3 \\
+/-63.3 \\
\end{array}$ & $\begin{array}{c}22.9 \\
+/-6.9\end{array}$ \\
\hline 6 & 3.7 & $\begin{array}{c}98.2 \\
+/-20.4 \\
\end{array}$ & $\begin{array}{c}138.4 \\
+/-27.2 \\
\end{array}$ & $\begin{array}{c}67.1 \\
+/-19.6 \\
\end{array}$ & $\begin{array}{c}23.8 \\
+/-7.1 \\
\end{array}$ \\
\hline 7 & 2.5 & $\begin{array}{c}95.2 \\
+/-24.3\end{array}$ & $\begin{array}{c}133.5 \\
+/-29.4\end{array}$ & $\begin{array}{c}65.9 \\
+/-17.9\end{array}$ & $\begin{array}{c}24.0 \\
+/-8.1\end{array}$ \\
\hline
\end{tabular}

Table 2 Evaluation of the average and Std values for the physiologic values at different Riker classes

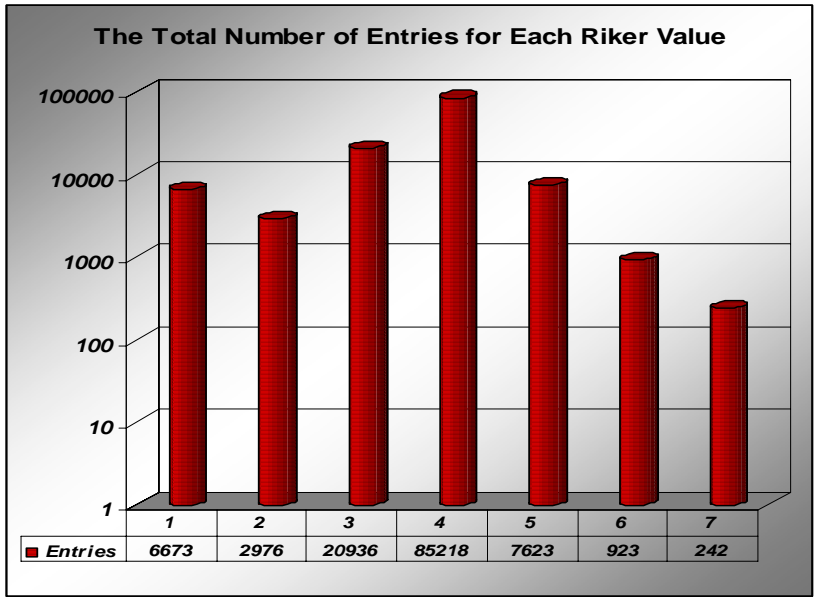

Figure 2 Total number of entries for each Riker Class (Value)

The mean value and standard deviation (Std) for each Riker class was calculated for the entire database (Table 2). The mean and standard deviation for HR, BP, and RR at each Riker class is shown in Figures 3, 4, and 5. For both the mean and standard deviation, there seems to be a gradual increase in HR, BP, and RR with an increase in agitation. Unarousable patients (Riker class 1) seem to have a slightly increased $\mathrm{HR}, \mathrm{BP}$, and $\mathrm{RR}$ when compared to the very sedate patients (Riker class 2).

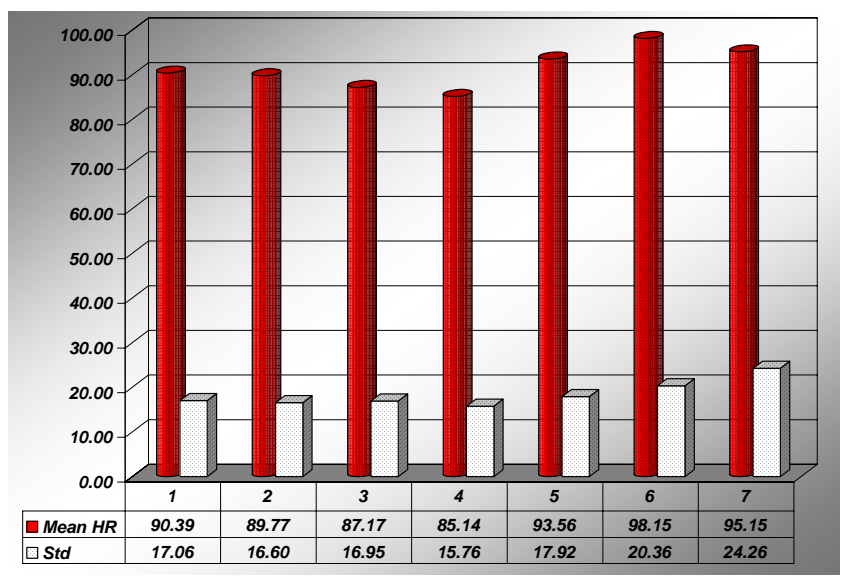

Figure 3 The mean and standard deviation for the heart rate at each Riker value 


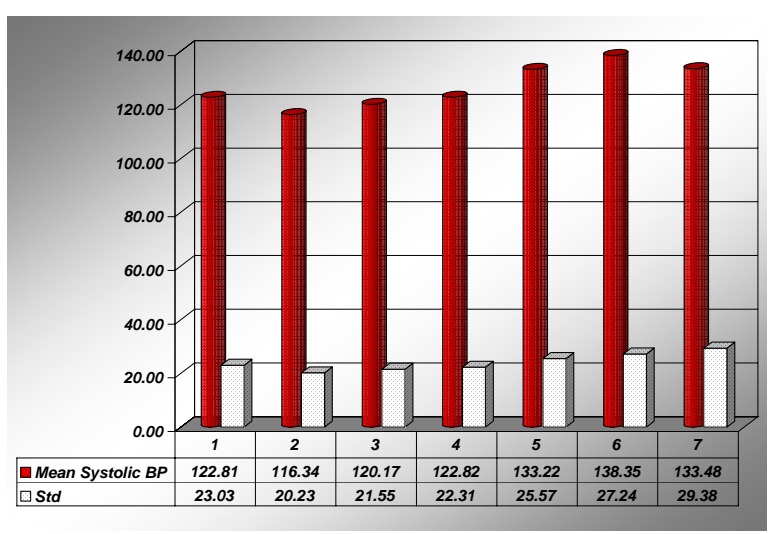

Figure 4 The mean and standard deviation for the systolic blood pressure at each Riker value

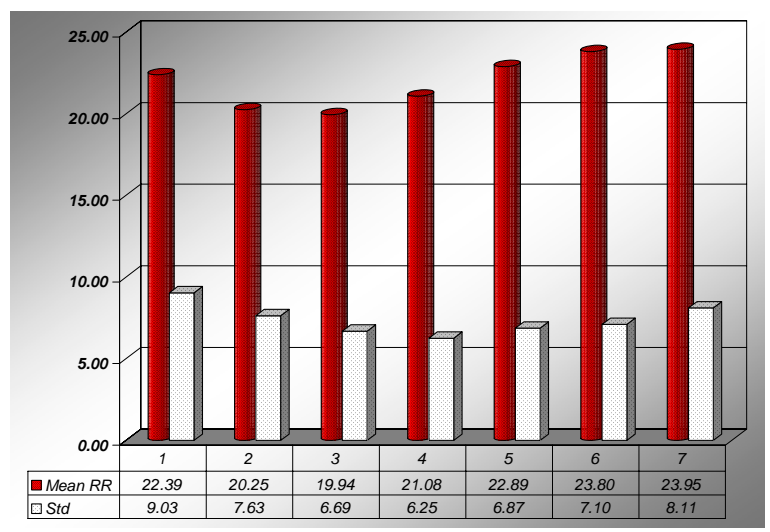

Figure 5 The mean and standard deviation for the respiratory rate at each Riker value

\begin{tabular}{|c|c|c|c|c|}
\hline 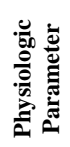 & 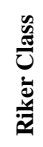 & 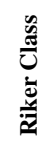 & 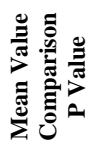 & 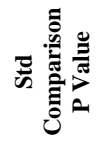 \\
\hline \multirow{3}{*}{ 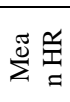 } & 1 & 4 & $<0.05$ & $<0.05$ \\
\hline & 1 & 7 & $<0.05$ & $<0.05$ \\
\hline & 4 & 7 & $<0.05$ & $<0.05$ \\
\hline \multirow{3}{*}{ 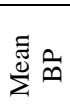 } & 1 & 4 & $<0.05$ & $<0.05$ \\
\hline & 1 & 7 & $<0.05$ & $<0.05$ \\
\hline & 4 & 7 & $<0.05$ & $<0.05$ \\
\hline \multirow{3}{*}{ 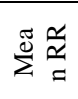 } & 1 & 4 & $<0.05$ & $<0.05$ \\
\hline & 1 & 7 & $<0.05$ & $<0.05$ \\
\hline & 4 & 7 & $<0.05$ & $<0.05$ \\
\hline
\end{tabular}

Table 3 Statistical analysis for different Riker classes using the mean and std for HR, BP, and RR

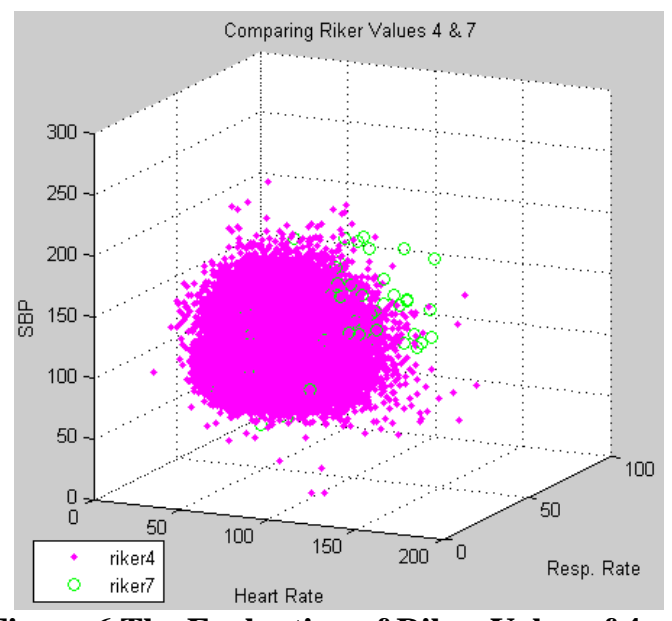

Figure 6 The Evaluation of Riker Value of 4 and 7 with Diastolic BP and HR

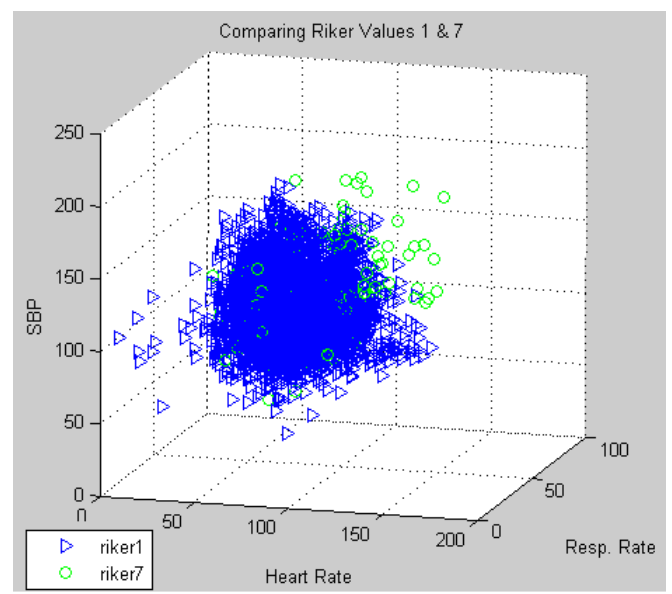

Figure 7 The Evaluation of Riker Value 1 and 7 with Diastolic BP and HR

A standard student's t-test for unequal variances was performed to compare each individual value between Riker classes. (The assumption of temporal independence between values is reasonable as Riker scores were recorded at intervals of several hours). The results of the t-test are listed in Table 3 for the mean values and standard deviations. An F-test was also applied to evaluate for inter variance differences between classes, which also demonstrate significant inter-class variance differences. Figures 6 and 7 illustrate the empirical estimates of the joint probability density functions (PDFs) for BP, HR, and RR at different Riker classes.

The ECG derived noise metric (number of unclassified beats per minute) was used to evaluate 5 patients in the ICU that were described as either sedate or severely agitated. Figures 8 \& 9 show the Riker scale and nonclassified beats for one patient. The ECG derived noise metric is based on the number of non-classified beats recorded over a period of time. 


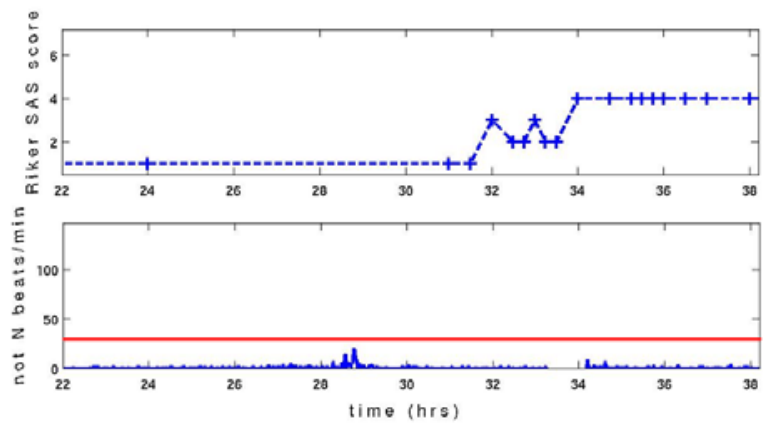

Figure 8 Non-classified beats for a Riker scale between 1 and 4 in an ICU patient

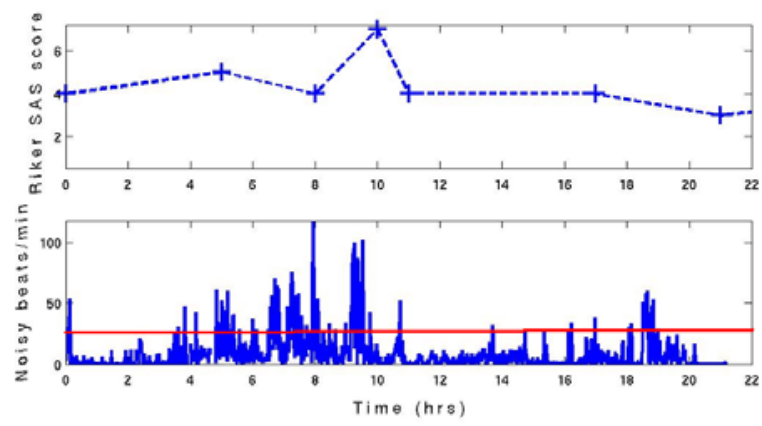

Figure 9 Non-classified beats for a Riker scale between 4 and 7 in an ICU patient

\section{Discussion and Conclusions}

Excessive agitation and sedation in the ICU can prolong recovery, can interfere with the administration of drugs, and decrease the safety of the patient and staff [2]. Although a statistical difference can be seen between the different Riker classes using HR, BP, and RR, these differences are not sufficient to differentiate individuals in any particular class (See Figures 6 \& 7).

The BP, HR, and BP show a gradual rise in values and standard deviation as a patient becomes more agitated (Figures 3, 4, 5) but there is also a slight increase in these parameters when the patient becomes unarousable (Riker class 1 ). It is possible that patients who are more heavily sedated are also more critically ill leading to an increase in variability for the HR, BP and RR [6]. The changes in $\mathrm{BP}, \mathrm{HR}$, and RR between Riker classes are not significant enough to have a major impact on a derived metric to quantitatively separate sedation from agitation.

The ECG-derived noise metric to classify agitated patients does show some promise. The assumption when using the noise metric is that an agitated patient will have an increased noisy ECG signals due to increased activity. A sedate patient or calm patient will have a lower number of noisy, non-classified, ECG beats compared to an agitated patient. A patient that is agitated will tend to have a noisier ECG strip over that period of time.

The five patients that were evaluated for this study had with Riker values ranging from 1 to 7 . The total number of noisy beats per minute was less than twenty-five $94 \%$ of the time when the patients were either sedate or calm, Riker class 1 to 4 (Figure 7). When the patients became agitated, Riker class 5 \& 7, the number non-classified ECG beats increased to greater than twenty-five $92 \%$ of the time. The ECG-derived noise metric might help to automatically detect the activity of a patient and whether that patient is agitated. There is an increasing need for a better-automated metric to evaluate sleep, sedation, and agitation in the ICU. Future work in this area will look at coupling an EEG and/or Bispectral Index (BIS) monitor with an ECG-derived noise metric to better quantify sleep, sedation, agitation, and activity in the ICU.

\section{Acknowledgements}

This publication was made possible by Grant Number R01-EB001659 from the National Institute of Biomedical Imaging and Bioengineering.

\section{References}

[1] Riker, R.R., J.T. Picard, and G.L. Fraser, Prospective evaluation of the Sedation-Agitation Scale for adult critically ill patients. Crit Care Med, 1999. 27(7): p. 1325-9.

[2] Chase, J.G., et al., Quantifying agitation in sedated ICU patients using heart rate and blood pressure. Physiol Meas, 2004. 25(4): p. 1037-51.

[3] Chase, J.G., et al., Quantifying agitation in sedated ICU patients using digital imaging. Comput Methods Programs Biomed, 2004. 76(2): p. 131-41.

[4] Saeed, M., et al., MIMIC II: a massive temporal ICU patient database to support research in intelligent patient monitoring. Comput Cardiol, 2002. 29: p. 641-4.

[5] Clifford G.D., M.P.E., Tarassenko L., Characterizing Artefact in the Normal Human 24-Hour RR Time Series to Aid Identification and Artificial Replication of Circadian Variations in Human Beat to Beat Heart Rate Using a Simple Threshold. Computers in Cardiology, 2002(29): p. 129-132.

[6] Simmons, L.E., et al., Assessing sedation during intensive care unit mechanical ventilation with the Bispectral Index and the Sedation-Agitation Scale. Crit Care Med, 1999. 27(8): p. 1499-504.

Address for correspondence:

Brian A. Janz MD bjanz@mit.edu

Harvard-MIT Division of Health Sciences \& Technology

Rm E25-505, 45 Carleton St., Cambridge MA 02142 USA 